\title{
The Impacts of the COVID-19 Pandemic on Liver Transplant Patients; Time to Change the Priorities
}

\author{
Arash Dooghaie Moghadam, MD'; Pegah Eslami, MD; Niloofar Razavi-Khorasani, MD²; Bobak Moazzami, MD²; Mostafa Mousavizadeh, \\ $\mathrm{MD}^{3}$; Mohammad Reza Zali, MD ${ }^{*}$; Amir Sadeghi, $\mathrm{MD}^{1^{*}}$ \\ ${ }^{1}$ Gastroenterology and Liver Diseases Research Center, Research Institute for Gastroenterology and Liver Diseases, Shahid Beheshti University \\ of Medical Sciences, Tehran, Iran \\ ${ }^{2}$ Network of Immunity in Infection, Malignancy and Autoimmunity (NIIMA), Universal Scientific Education and Research Network (USERN), \\ Tehran, Iran \\ ${ }^{3}$ Heart Valve Disease Research Center, Rajaie Cardiovascular Medical and Research Center, Tehran, Iran
}

\section{Dear Editor,}

The current coronavirus disease 2019 (COVID-19) crisis has emerged as a global pandemic. ${ }^{1,2}$ Iran has been widely exposed to the COVID-19 and has the fifth highest death toll from COVID-19. The Taleghani Liver Transplant Center, one of the high-volume liver transplant centers in the Middle East, has provided new guidelines for its ward and attempted to provide an appropriate model for these patients based on previous pandemic studies. According to the published studies, the first experience with a pandemic in the transplantation era was due to $\mathrm{H} 5 \mathrm{~N} 1$ pandemic. ${ }^{3}$ In addition, the information on the influenza virus reminds us that transplanted patients are likely to constitute a larger number of symptomatic patients due to immunosuppression, if they are exposed to the pathogen and the disease.

An Italian study showed that $20 \%$ of healthcare workers were infected with the new coronavirus and some of them died. ${ }^{4}$ Therefore, in order to minimize the risk of healthcare providers' exposure and reducing patients' flow to hospitals, telemedicine could be used as a cheap and effective method for educating patients in developing countries. ${ }^{5}$ Patients should be trained about the risks of attending public centers and disease prevention, considering the high burden of liver transplantation and its heavy costs to health systems. Therefore, specific training should be provided on the Internet and webbased platforms for these patients. They should inform their transplant physicians before any referral to the health centers for a preliminary evaluation of symptoms and provide a complete list of their medications and their transplant records.

Previously, it has been demonstrated that higher virus levels could be found in the transplanted tissue after death when compared to the non-transplanted individuals. ${ }^{6}$ Moreover, in a study by Al-Ghamdi et al, unfavorable outcomes were more frequently reported for transplanted individuals in the MERS-CoV virus. ${ }^{7}$ Therefore, the Taleghani Center decided to focus on pandemic prevention of post-transplant infection and limit its surgical operations to emergency surgery considering the low probable outcome. Furthermore, owing to the pandemic nature of this disease and the need for ICU beds, it is recommended to avoid over-hospitalization and elective surgery, to consider isolated places for the patients' follow-up in the early days, and to conduct patients' follow-up and referrals through phone calls or clinically outside the hospital.

Another aspect highly considered in liver transplant patients is the mental health status of these patients associated with the poorer transplantation outcome and inappropriate drug consumption by the patients. Regarding COVID 19, Xiang showed that like other pandemics, there is a growing concern about the death of patients and quarantined people. ${ }^{8}$ A study by Bao also reported stress was caused by uncertainty about the condition and the exact time of disease control. ${ }^{9}$ Thus, they predicted a higher risk of hopelessness and anxiety owing to chronic disease in transplanted patients. Undoubtedly, these patients should undergo psychiatric telephone counseling during the crisis for psychological assessments and stress reduction techniques, home exercise activities, and proper nutrition. Moreover, Wang et al showed that lower family income was associated with a lower rate of health-related quality of life. ${ }^{10}$ Accordingly, during this pandemic, financial support for these patients from the government or other foundations presumably improve their quality of life.

Re-transplantation is also an important factor associated

*Corresponding Authors: Amir Sadeghi, MD; Gastroenterology and Liver Diseases Research Center, Research Institute for Gastroenterology and Liver Diseases, Shahid Beheshti University of Medical Sciences, Tehran, Iran. Tel: +98-21-22432525; Fax: +98-21-22432517; Email: amirsadeghimd188@gmail.com. Mohammadreza Zali, MD; Gastroenterology and Liver Diseases Research Center, Research Institute for Gastroenterology and Liver Diseases, Shahid Beheshti University of Medical Sciences, Tehran, Iran. Tel: +98-21-22432525 Fax: +98-21-22432517. Email: Itrc.tehran@gmail.com 
with a dramatic loss of quality of life. ${ }^{11}$ Therefore, this group of patients need greater attention during this pandemic. Transplant candidates encounter a significant range of stressors before their transplantation ${ }^{12}$; thus, they should also be reassured about receiving their transplants after the disease is controlled and the crisis is resolved. According to Banihashemi et al, a healthy lifestyle, financial support and treatment of major psychiatric disorders improve the quality of life in Iranian liver transplant patients. ${ }^{13}$ Hence, it is recommended to provide psychiatric services first to patients with lower MELD scores and to those who have already undergone transplantation due to the limited financial resources in the health system. Overall, it can be concluded that offering special support is necessary for transplant candidates. The patients' access to their medication is another problem in developing countries during this pandemic. It is recommended to supply these medications to the nearest local health center during the crisis and deliver them to the patients after approval by the Transplant Center.

It should be noted that transplant patients likely infected with the new coronavirus are treated with common relevant protocols; hence, appropriate measures should be taken into consideration regarding training physicians about the possible drug interactions with transplant patients' medications.

\section{Authors' Contribution}

ADM and PE: designing of the research. BM and NRK: drafting the manuscript. MM, MRZ and AS: drafting and finalizing the manuscript.

\section{Conflict of Interest Disclosures \\ None}

\section{Ethical Statement}

Not applicable.

\section{References}

1. Zhu N, Zhang D, Wang W, Li X, Yang B, Song J, et al. A Novel Coronavirus from Patients with Pneumonia in China, 2019. N Engl J Med. 2020;382(8):727-33. doi: 10.1056/
NEJMoa2001017

2. Cucinotta D, Vanelli M. WHO Declares COVID-19 a Pandemic. Acta Biomed. 2020;91(1):157-60. doi: 10.23750/ abm.v91i1.9397.

3. Kumar D, Humar A. Pandemic influenza and its implications for transplantation. Am J Transplant. 2006;6(7):1512-7. doi: 10.1111/j.1600-6143.2006.01364.x.

4. The Lancet. COVID-19: protecting health-care workers. Lancet. 2020;395(10228):922. doi: 10.1016/S01406736(20)30644-9.

5. Moazzami B, Razavi-Khorasani N, Dooghaie Moghadam A, Farokhi E, Rezaei N. COVID-19 and Telemedicine: Immediate action required for maintaining healthcare providers well-being. J Clin Virol. 2020:104345. doi: 10.1016/j. jcv.2020.104345.

6. Kumar D, Humar A. Emerging viral infections in transplant recipients. Curr Opin Infect Dis. 2005;18(4):337-41. doi: 10.1097/01.qco.0000172697.44784.ff.

7. Al Ghamdi M, Alghamdi KM, Ghandoora Y, Alzahrani A, Salah F, Alsulami A, et al. Treatment outcomes for patients with Middle Eastern Respiratory Syndrome Coronavirus (MERS $\mathrm{CoV}$ ) infection at a coronavirus referral center in the Kingdom of Saudi Arabia. BMC Infect Dis. 2016;16:174. doi: 10.1186/ s12879-016-1492-4.

8. Xiang YT, Yang Y, Li W, Zhang L, Zhang Q, Cheung T, et al. Timely mental health care for the 2019 novel coronavirus outbreak is urgently needed. Lancet Psychiatry. 2020;7(3):2289. doi: 10.1016/S2215-0366(20)30046-8.

9. Bao Y, Sun Y, Meng S, Shi J, Lu L. 2019-nCoV epidemic: address mental health care to empower society. Lancet. 2020;395(10224):e37-e8. doi: 10.1016/S01406736(20)30309-3.

10. Wang GS, Yang Y, Li H, Jiang N, Fu BS, Jin H, et al. Healthrelated quality of life after liver transplantation: the experience from a single Chinese center. Hepatobiliary Pancreat Dis Int. 2012;11(3):262-6. doi: 10.1016/s1499-3872(12)60158-1.

11. Braun F, Teren K, Wilms P, Gunther R, Allmann J, Broering DC, et al. Quality of life after liver transplantation. Transplant Proc. 2009;41(6):2564-6. doi:10.1016/j.transproceed.2009.06.030.

12. Rosenberger EM, Dew MA, DiMartini AF, DeVito Dabbs AJ, Yusen RD. Psychosocial issues facing lung transplant candidates, recipients and family caregivers. Thorac Surg Clin. 2012;22(4):517-29. doi: 10.1016/j.thorsurg.2012.08.001.

13. Banihashemi $M$, Hafezi $M$, Nasiri-Toosi $M$, Jafarian A, Abbasi MR, Arbabi M, et al. Psychosocial Status of Liver Transplant Candidates in Iran and Its Correlation with Health-Related Quality of Life and Depression and Anxiety. J Transplant. 2015;2015:329615. doi: 10.1155/2015/329615.

Cite this article as: Dooghaie Moghadam A, Eslami P, Razavi-Khorasani N, Moazzami B, Mousavizadeh M, Zali MR, et al. The impacts of the COVID-19 pandemic on liver transplant patients; time to change the priorities. Arch Iran Med. 2020;23(7):507-508. doi: 10.34172/aim.2020.50.

(c) () () 2020 The Author(s). This is an open-access article distributed under the terms of the Creative Commons Attribution License (http://creativecommons. org/licenses/by/4.0), which permits unrestricted use, distribution, and reproduction in any medium, provided the original work is properly cited. 